\title{
Adultos mayores en funciones de cuidadores de ancianos
}

\section{Older adults as caregivers for aged persons}

\author{
MsC. J uan Carlos Baster Moro \\ Universidad de Ciencias Médicas de Holguín. Holguín, Cuba.
}

\section{RESUMEN}

Se realizó una revisión que aborda el envejecimiento como comportamiento demográfico, como proceso y la vejez como edad de la vida que puede ser una etapa de desarrollo, pero que puede estar caracterizada por la necesidad de recibir cuidados, que varían en dependencia de las condiciones biomédicas de la persona. Los cuidados son ofrecidos en muchos casos por personas envejecidas también, situación en que se describen problemas que hacen compleja la acción de cuidar. Es vital la preparación y atención a cuidadores y familias de ancianos. Se describe el desarrollo de instituciones para ofrecer cuidados cuando definitivamente no existe familia.

Palabras clave: Adulto mayor, envejecimiento, cuidadores, familia.

\section{ABSTRACT}

A review was made on aging as demographic behavior and as a process; the old age as a phase of life may be a development phase, but it can also be characterized by the need of being cared for, depending on the biomedical conditions of that person. Care is often provided by people who are also elderly persons, which sometimes leads to problems adding more complexity to the care-giving function. It is vital to prepare and pay attention to the caregivers and the relatives of the elderly. The development of institutions was described to offer care when there is no family at all.

Key words: Older adult, aging, caregivers, family. 


\section{NTRODUCCIÓN}

La definición de envejecimiento poblacional establece como tal, el aumento de la proporción de personas de edad avanzada en relación con el resto de la población, sin embargo, se ha considerado la importancia de definirla también como la inversión de la pirámide de edades, debido a que el fenómeno, no es solamente un aumento de la proporción de adultos mayores, personas de 60 años o más, ${ }^{1}$ es también una disminución de la proporción de niños y jóvenes menores de 15 años. Este último es el elemento clave que caracteriza al envejecimiento poblacional. ${ }^{2,3}$

Las edades avanzadas de la vida están llenas de riquezas propias, que todos los seres humanos pueden disfrutar, es una etapa en que hay capacidades a fortalecerse y otras por desarrollarse; en ese sentido, uno de los mayores esfuerzos debe estar encaminado a la adquisición de conductas individuales que haga a estas personas más saludables e independientes, por ejemplo, mediante el proceso educativo los ancianos pueden incorporar a su vida diaria conductas de salud física, social, mental y espiritual que favorezcan su estado de bienestar. ${ }^{4}$

En sentido contrario, está el hecho de necesitar cuidados, conjunto de actividades humanas, físicas, mentales y emocionales dirigidas a mantener la salud y el bienestar de individuos o comunidad. Comprende, entre otras, mantener una alimentación adecuada, hábitos de higiene corporal y del hogar, evitación de posibles peligros o accidentes, sin olvidar la atención emocional y afectiva, las relaciones sociales y el entretenimiento. ${ }^{5}$

Las condiciones físicas y mentales diferencian los cuidados a realizar en los adultos mayores, no es lo mismo que el anciano que se cuida deambule y conserve aunque sea el mínimo de independencia para las actividades básicas de la vida diaria, a que esté en estado de postración, en el cual hay que ejecutarles toda la asistencia, cuya diversidad estará mediada por el estado físico y puede ir en incremento, como por ejemplo, alimentación por levine, incontinencia de esfínter vesical y anal, escaras, rigidez, flacidez u otros. Las condiciones mentales también median los cuidados, la demencia puede ser un gran problema geriátrico que exige de los cuidadores un nivel superior de actuación y comprensión, y es necesario saber que esta enfermedad puede ir también en aumento y crear las más disímiles reacciones en los familiares ${ }^{6,7}$ que pueden no comprender lo que sucede, que su familiar ya no es el mismo ni tiene un comportamiento remotamente adecuado dentro del ámbito familiar y social.

La necesaria incorporación social de los miembros más jóvenes de las familias, favorece que en aquellas con miembros envejecidos con necesidad de cuidados, estos sean asumidos en muchos casos por otros miembros adultos mayores o también, de diversos parentesco como esposa o esposa, hermanos e incluso hijos que rebasan la barrera cronológica de los 60 años, pero en condiciones diferentes de salud.

En Cuba, a pesar del predominio de familias extensa y ampliadas, existen otras en que los mayores viven solos, alejados de hijos y nietos, que necesitan cuidados de forma intermitente y estos recaen igualmente en adultos mayores de esa familia nuclear. 
Algunas de las condiciones que complican los cuidados de ancianos por ancianos, se relacionan a continuación:

- Cambios propios del envejecimiento en ambos.

- Enfermedades asociadas.

- Deterioro cognitivo.

- Limitaciones crecientes para las actividades instrumentadas y básicas de la vida diaria.

- Dificultades para el manejo de la prescripción médica.

- Necesidad del uso de ayudas técnicas.

- Carencia de camas fowler, colchones antiescaras y sillas de ruedas acordes a la edad y que faciliten los cuidados.

- Viviendas con barreras arquitectónicas.

- Dificultades para la compra y elaboración de los alimentos.

- Vestidos y calzados no adecuados para la edad.

- Dificultades para el traslado a unidades asistenciales por carencia de trasporte sanitario u otro adecuado a las condiciones del paciente.

- Limitaciones económicas.

- Estereotipos distorsionados en vecinos y comunidad hacia los mayores.

- Las opciones de funcionamiento diurno no cubren todas las necesidades y su variabilidad es aún limitada.

El desempeño de un adulto mayor en funciones de cuidador ${ }^{5}$ exige de este una entrega para la cual sus capacidades también están envejecidas, y están presentes en él enfermedades o limitaciones que tienden a incrementarse al ofrecer cuidados, y hacen que se sumen a la ya existente sobrecarga del cuidador. ${ }^{8}$

La carga sanitaria en estos casos es mayor porque las orientaciones médicas deben brindarse con absoluta claridad y verificarse con frecuencia, las visitas del Equipo Básico de Salud (EBS) y de Equipo Multidisciplinario de Atención Gerontológica (EMAG) deben hacerse más asiduas, las intervenciones de salud en este medio pueden demandar material de curación, procesos de atención de enfermería, ayudas técnicas, entre otras, dentro de la propia vivienda. El ingreso en el hogar puede ser en estos casos una de las actividades específicas de la medicina general integral a explotar para beneficio del paciente, del cuidador, de la familia y del sistema sanitario. 
A juicio de este autor se hace pertinente la preparación de las familias para asumir los cuidados de sus miembros adultos mayores independientemente de las condiciones en que estos se encuentren.

En los casos carentes de familiares responsables para los cuidados intradomiciliarios y después de haber agotados todas las posibilidades comunitarias, será necesario considerar la institucionalización, la entrada o admisión a un hogar de ancianos, que debiera ser temporal pero en la mayoría de los casos las circunstancias que impusieron tal decisión se mantienen en el tiempo, y por lo tanto el retorno al medio será imposible, en otros casos el estado mórbido del paciente hace que la institución sea su última residencia hasta fallecer.

Al cierre de 2010 la cifra de adultos mayores cubanos ascendía a 1996 632, 17,8 $\%$ de envejecimiento, con una esperanza de vida al nacer en 77,97, mientras la geriátrica (a los 60 años) está estimada en 22,09 años, ${ }^{9}$ esta realidad plantea que los miembros de esa edad continuarán en franco incremento; las proyecciones demográficas plantean que en el 2030 tendremos 3,4 millones de personas seniles, $31 \%$, el país más envejecido de América Latina y el Caribe, cifra que se espera se eleve a 38 \% según cálculos de Naciones Unidas, ubicándonos en el lugar 11 entre los estados más envejecidos. ${ }^{10}$

En Cuba, debido al creciente envejecimiento poblacional y la extensión de la vida hasta edades muy avanzadas, con la consiguiente asociación de disminución de funciones, comorbilidad y discapacidad, deben prevalecer las llamadas opciones de funcionamiento diurno y diversificarlas en tan variadas tipologías como situaciones medicosociales se puedan presentar. Es necesario incrementar las ayudas técnicas para el hogar, que contribuyan a conservar las reservas funcionales de gerontes y facilitar su cuidado para que su validismo permita a la familia continuar con su función social libre de preocupaciones por los riesgos de los ancianos a cuidar. Debe, además, desarrollarse en el país propuestas de intervención para el apoyo al cuidador. ${ }^{11-13}$

El mundo lleva cabo un proyecto global denominado "Ciudades Amigables con la Edad", auspiciado por la Organización Mundial de la Salud, que prevé entre sus muy diversas propuestas los cuidados domiciliarios, ${ }^{14}$ que pasan por diferentes modalidades y toman las más variadas formas, según culturas, tipo de sociedad, concepciones religiosas, entre otras.

\section{REFERENCI AS BI BLI OGRÁFICAS}

1. INFOGER-2001 [Internet]. Convocatoria de evento. Cuba: INFOGER; 2001 [citado 20 Ago 2010]. Disponible en: http://www.ccram.sld.cu/infoger/infoger\%202001/programin2001.htm

2. Chávez Negrin E. Algunas consideraciones sobre el envejecimiento demográfico en Cuba [Internet]. En: Taller Población y desarrollo. La Habana: CEDEM; Jul 2002 [citado 20 Ago 2010]. Disponible en: http://bibliotecavirtual. clacso.org.ar/ar/libros/cuba/cips/caudales05/Caudales/ARTI CULOS/ArticulosPDF/1819C020.pdf 
3. Baster Moro JC. Atención médico social al adulto mayor en la provincia Holguín. Rev Cubana Salud Pública [Internet]. 2011[citado 23 sept 2011];37(3). Disponible en: http://scielo.sld.cu/scielo.php?script=sci_arttext\&pid=S086434662011000300004\&lng=es\&nrm=iso\&tlng=es

4. Carrasco García MR. Usted puede... lograr una buena longevidad. La Habana: Editorial Científico-Técnica; 2005.

5. Baster Moro JC. Glosario de Términos y Definiciones de Gerontología y Geriatría. La Habana: Editorial Ciencias Médicas; 2011.

6. Margarida Sotto Mayor M, Ribeiro O, Paúl C. Satisfaction in Dementia and Stroke Caregivers: A Comparative Study. Rev Latinoamer Enfermagem [Internet]. 2009 [citado 8 Sept 2011];17(5):620-4. Available from: http://ead.eerp.usp. br/rlae/

7. Pérez Martínez VT, de la Vega Pazitková T. Repercusión de la demencia en los cuidadores primordiales del policlínico "Ana Betancourt". Rev Cubana de Med Gen Integr [Internet]. 2010 [citado 1 Sept 2011];26(2):215-24. Disponible en: http://scielo.sld.cu/scielo.php?script=sci arttext\&pid=S0864-

$\underline{21252010000200003 \& \operatorname{lng}=e s \& n r m=i s o \& \operatorname{ting}=e s}$

8. Lluch Bonet A, Morales López A, Cabrera Adán M, Betancourt Navarro M. Factores previsibles en la salud física y psicosocial del cuidador crucial del anciano con demencia en el hogar. Rev Cubana Enfermer [Internet]. 2010[citado 1 Sept 2011]; 26(2): 3-13. Disponible en:

http://scielo.sld.cu/scielo.php?script=sci arttext\&pid=S0864-

$03192010000200002 \& \operatorname{lng}=\mathrm{es \& nrm=iso \& \operatorname {lng } = e s}$

9. Centro de Estudios de Población y Desarrollo. El envejecimiento de la población. Cuba y su territorios [Internet]. 2010 [citado 1 Sept 2011]. Disponible en: http://www.one.cu/envejecimiento2010.htm

10. Oficina Nacional de Estadísticas. Proyecciones de la Población Cubana 20102030 [Internet]. 20101 [citado 10 Sept 2011]. Disponible en: http:// www. one.cu/proyecciones $\% 20$ de $\% 201$ a $\% 2$ poblacion $\% 202010 \% 202030$ .htm

11. Espín Andrade. "Escuela de Cuidadores" como programa psicoeducativo para cuidadores informales de adultos mayores con demencia. Rev Cubana Salud Pública [Internet]. 2009 [citado 23 sept 2011];35(2). Disponible en: http://scielo.sld.cu/scielo.php?script=sci arttext\&pid=S0864 $\underline{34662009000200019 \& \operatorname{lng}=\mathrm{es} \& \mathrm{nrm}=\mathrm{iso \& t} \operatorname{lng}=\mathrm{es}}$

12. Pérez Perdomo M. Las intervenciones dirigidas a los cuidadores de adultos mayores con enfermedad de Alzheimer. Rev Habanera Cienc Méd [Internet]. 2008 [citado 23 sept 2011]; 7(3). Disponible en: http://scielo.sld.cu/scielo. php?script=sci arttext\&pid=S1729 $\underline{519 \times 2008000300009 \& \operatorname{lng}=\mathrm{es} \& n r m=\mathrm{iso} / \mathbb{\&} \mathrm{t} \operatorname{lng}=\mathrm{es}}$ 
13. Alcaraz Agüero M, Fong Estrada A, Laborí Ruiz JR, Alayo Blanco M, García Álvarez R. Capacitación a personas cuidadoras de pacientes con demencia. MEDISAN [Internet]. 2009 [citado 23 sept 2011];13(2). Disponible en: http://scielo.sld.cu/scielo.php? script=sci arttext\&pid=S1029-

30192009000200004\&lng=es\&nrm=iso\&itlng=es

14. Organización Mundial de la Salud. Ciudades Globales Amigables con los mayores: una Guía. Ginebra: OMS; 2007.

Recibido: 11 de octubre de 2011.

Aprobado: 28 de octubre de 2011.

Juan Carlos Baster Moro. Calle Maceo No. 244 e/ Cables y Ángel Guerra. Holguín 80100, Cuba.

Telf.: 42-9044. Correo electrónico: juancarlosbm@infomed.sld.cu 